in the Grimms' Collection Can Foster Social Integrative Behaviours in Children”, penned by María Alcantud Díaz, considers reading literature, particularly fairy tales, in light of key competences for lifelong learning suggested by the European Union.

As the editors state in the introduction, all twelve chapters share the goal of materialising the full potential that is set off when children's literature takes the central place within the educational endeavour. A variety of perspectives and ideas is included and elaborated by scholars from across Europe: Spain, England, Italy, Sweden and Greece. All the contributions are well referenced and some of them contain illustrations, charts and pictures, as well as questions that can trigger discussion and serve as starting points for further research. This book offers a fresh and inspiring view of some key concepts in the study of children's literature and language teaching, and introduces major trends and innovations that characterise it. This engaging and useful collection is of interest not only to scholars, teachers, teacher trainees, but also to both the general public and academic readers interested in this field of study.

Dina Alexandra Pavković

\title{
Neue Einblicke in die Produktion der russischen Exilkinderliteratur
}

Nadia Preindl. 2016. Russische Kinderliteratur im europäischen Exil der Zwischenkriegszeit. Frankfurt am Main-Bern-Bruxelles-New York-Oxford, Warszawa-Wien: Peter Lang (Russian Culture in Europe 11). 278 Seiten. ISBN 978-3-631-67020-0 (Print). 978-3-653-06179-6 (E-Book). DOI 10.3726/978-3653-06179-6

DOI: 10.21066/carcl.libri.2016-05(02).0017

Der vorliegende Band ist die überarbeitete Fassung von Nadia Preindls 2014 am Institut für Slawistik der Universität Wien bei Fedor Poljakov eingereichter Dissertation „Russische Kinderliteratur im europäischen Exil zwischen 1918 und 1939“. Die Monographie bietet nach einer Einführung zu Forschungslage, literaturgeschichtlichem Kontext und Kindheit im Exil mit Schwerpunkt auf der Rolle des Kinderbuches zunächst eine Beschreibung und Typologisierung der (vielgestaltigen) Quellen, gefolgt von theoretischen Diskussionen zu Kinderliteratur im Spiegel pädagogischer und literarischer Kritik, und wird stimmig abgeschlossen mit vier Einzelanalysen. Das breite Resümee ist dreisprachig (deutsch, russisch und englisch). Dazu tritt ein reiches, nach Publikationsformen gegliedertes Quellenkorpus inklusive zahlreicher Abbildungen von Titelblättern und dergleichen mehr, die eindrucksvoll die künstlerische Hochwertigkeit und liebevolle Gestaltung der Veröffentlichungen zeigen. (Ein kleines Manko ist, dass corpus bei Preindl durchgehend masculini generis ist - ein Lapsus, der die Rezensentin vielleicht nur aufgrund ihres latinistischen Hintergrundes stört; deren komparatistischer Background sichert der Verfasserin jedoch die Anerkennung für die Kombination von großer Übersicht und Gespür für aussagekräftige Schwerpunktsetzung.) Das Verzeichnis enthält neben Veröffentlichungen, die als kindgerecht eingestuft wurden, ohne Kinder- oder Jugendliteratur zu sein, sämtliche Werke für Kinder und Jugendliche, die in der Zwischenkriegszeit in den Exilzentren Europas, aber auch in Berlin, für den russischen Markt gedruckt wurden. Ein umfangreicher Anhang mit Werkverzeichnissen in 
ausgewählten Periodika, eine informative Bibliographie, ein Abbildungsverzeichnis und ein hilfreiches Personenregister runden das Buch ab.

Durch Preindls verdienstvolle Studie zieht sich wie ein roter Faden das Bedürfnis der vorgestellten Autoren und Herausgeber, den Heranwachsenden ein Stück russischer Heimat(bezogenheit) in ihrem neuen Lebensumfeld $\mathrm{zu}$ erhalten (oder wiederzugeben). Keineswegs unberechtigt befürchtete man Sprachverlust und Identitätswandel. Von besonderem Interesse sind überdies ideologisch-weltanschauliche Unterschiede zwischen der Literatur, die im mehrere Jahrzehnte umfassenden Untersuchungszeitraum in Russland (bzw. der Sowjetunion) und außerhalb auf den Buchmarkt kam. Preindl unterscheidet (beginnend bereits vor der Wende zum 20. Jahrhundert) drei große Strömungen bis zur Oktoberrevolution (sentimentalistisch-moralistisch, künstlerischrealistisch, modernistisch). Dabei rückt die Ästhetik zusehends gegenüber der Didaktik in den Vordergrund, und der Prozentsatz der Autoren, die nicht in erster Linie mit Kinderund Jugendliteratur in Verbindung gebracht werden, steigt. In Sowjetrussland ging nach den Wirren des Jahres 1917 mit dem Anstieg der klassenkämpferischen Ausrichtung der Literatur für die Jüngsten eine langlebige Märchen- und (Anti-)phantastikdebatte einher: Tier- und Abenteuergeschichten wurden als zu trivial, als zu gestrig empfunden. Im Exil erkannte man das ,Heile Welt'-Potential, das gerade diese Texte boten; parallel dazu wurde - durchaus als (Überlebens-)hilfe - die neue, oft harte und schwierige Lebensrealität der Flüchtlingskinder literarisch aufgearbeitet.

Berlin, Prag und Paris wurden $\mathrm{zu}$ den zentralen Erscheinungsorten russischer Exilliteratur für junge LeserInnen; später kamen Finnland, Lettland, Schweden, Serbien, Bulgarien und diverse fernöstliche Exilzentren hinzu. Namhafte Literaturkritiker und eigens eingerichtete Kommissionen erarbeiteten (theoretische und praxisorientierte) Standards und Qualitätskriterien; erklärtes Ziel war eine Art Revival vorrevolutionärer Texte, durchaus intendiert als möglicher Ersatz für bolschewistische Literaturproduktion, geschuldet aber auch der pragmatischen Tatsache, dass der Wiederabdruck bereits existierender Literatur in der Fremde bedeutend einfacher war als die Publikation neuer Werke. Die Diskussion, ob es überhaupt spezieller Kinder- und Jugendliteratur bedürfe, reicht zurück bis ins 19. Jahrhundert und fand auch in der Emigration und im Exil - Termini, die es gemäß des state of the art der Exil- respektive Emigrationsforschung recht streng zu unterscheiden gilt - rege Fortsetzung. Lesepsychologie und ästhetischer Anspruch wurden zu den bestimmenden Kriterien; im Rückgriff auf vorrevolutionäre Kinderliteratur erhielt humanistischer Realismus den Vorzug vor moralisierender Verniedlichung, Klassiker der sogenannten Allgemeinliteratur blieben stets zentral, da so eine Heranführung an das literarische Erbe Russlands ermöglicht werden sollte. Der ideale Kinderschriftsteller war einer, der das Grundprinzip des Crosswriting verfolgte: jemand, dessen Texte auch für Erwachsene anziehend waren. Die Kinderliteratur im und zum Exil erhob den Anspruch, vollwertige Literatur zu sein. Die Themenwahl ist auf die Lebensrealität der jungen LeserInnen abgestimmt und soll - neben dem inneren Bezug zur alten Heimat - wenigstens eine gewisse Erleichterung in der neuen bieten; die Verfasserin exemplifiziert dies an vier (recht unterschiedlichen) Beispielen: So ist (a) bei Aleksandr Jablonovskij (Die Abenteuer des Miša Šišmarev, 1921) ein Flüchtlingskind auf einem Schiff von den vielen Eindrücken zunächst überwältigt und fühlt sich von den Menschenmassen eingeengt. Letztlich lernt es die Multikulturalität zu schätzen, wenngleich 
der schrittweise Sprachwechsel (in diesem Fall vom Russischen zum Englischen) schwierig ist. Die eingestreuten, nicht übersetzten fremdsprachigen Wendungen sind für Preindl ein Indikator dafür, dass die potentiellen LeserInnen als mehrsprachig eingestuft wurden; meines Erachtens liegt darin aber auch ein gezieltes Mittel, um die jungen RezipientInnen an das eigene Empfinden in einer vergleichbaren Situation zu erinnern und zu zeigen, dass (und wie) man damit fertig werden kann. In jedem Fall wird Autobiographisches für Jablonovskij ist dies belegbar - zu einem wichtigen Gestaltungselement; die jungen LeserInnen werden als reife RezipientInnen gesehen, weswegen ihnen auch ein offenes, keineswegs gesichert positives Ende zugemutet wird.

Im Zuge der in Russland und später in der Sowjetunion dominanten Märchendebatte ergaben sich vielfältige Wechselbeziehungen zur deutschen Literatur - etwa über Übersetzungen und auf dem Weg von Klassikerbearbeitungen für Kinder. Dazu kamen Almanache, in denen (trotz der Anthropomorphismusdebatte) Tiere eine wesentliche Rolle spielen. Auch kinderbiblische Elemente fanden in reichem Maß Eingang - ein Vorgang, der in der Sowjetunion unmöglich gewesen wäre. Die oftmals kritisierte Phantastik, die mit dem Auftritten von Tieren einhergeht, wird (b) von Saša Černyj (d.i. Aleksandr Michailovič Glikberg) in Wunderbarer Sommer insofern abgemildert, als sie in eine Art Traum- oder Gegenwelt ausgelagert wird: Ein bedrohliches „Krokodil“ ist in Wahrheit ein Pudel, dessen harmloses Bellen das schlafende Kind im Traum falsch interpretiert hat. Zudem wird das Gespräch mit Tieren zum idealen Vehikel für Zweisprachigkeit: Der kleine Protagonist spricht in der französischen Emigration mit den dort heimischen Tieren Französisch, weil sie Russisch nicht verstehen würden. Hinzu tritt die Stadt-Land-Diskrepanz: Der Bub hat schon mehrere Wohnsitzwechsel hinter sich. Die in Paris massiv empfundene Außenseiterrolle erscheint auf dem Land weniger ausgeprägt: So dominiert in Wunderbarer Sommer die positive Evasion, in Černyjs Lyrik hingegen reale Tristesse, v.a. aber Einsamkeit. Effektvoll ist die Entscheidung des Autors zur „Abkehr von der ,Onkelhaftigkeit““ (145) durch den literarischen Kunstgriff, alles aus der Perspektive des Kindes zu erzählen. Dies gelang, wie Preindl betont, in besonderem Maß durch Bewahrung der Kindlichkeit auf Seiten des Autors. Resultat ist ein höchst gelungenes Stück Crossover-Literatur, da sich mindestens so viele erwachsene LeserInnen dafür begeistern konnten wie auch jene im Kindes- und Jugendalter.

International wird (c) Michail Osorgin nicht primär als Kinder- und Jugendliteraturautor wahrgenommen; vielleicht erklärt das auch das doch sehr spezielle Setting seiner Erzählung Über den persönlichen Beziehungen stehend (1929): Zwei Gymnasiasten, deren Hobby es ist, gemeinsam (und laut deklamierend) russische Klassiker zu lesen, geraten in Streit und stellen ihre Treffen ein. Doch die Liebe zur Literatur lässt sie den mit dem für junge Menschen typischen ,heiligen Ernst', mit dem das Zerwürfnis gepflegt wird, nicht lange durchhalten; letztlich stellen sie das große gemeinsame Ideal über ihre kleinlichen Differenzen und begraben ihre Zwistigkeiten. Neben dieser patriotischen Botschaft, die dem Einstehen für die russische Literatur zweifellos anhaftet, ist der Text in erster Linie durch eine Fülle intertextueller Referenzen bemerkenswert: Den LeserInnen in der Emigration und im Exil wird durch diese Texte im Text (zuzüglich Literaturkritik und Kanonisierung) ein konstitutives Element der russischen Kultur (nebst Stolz und Heimatgefühl) vermittelt. Das damit unweigerlich verbundene Pathos dürfte Preindl so ergriffen haben, dass ihr in 
diesem Abschnitt einige Wiederholungen unterlaufen sind, die der zentralen und wichtigen Botschaft dieses Kapitels aber keinen Abbruch tun.

Ebenfalls markant ist (d) das vierte und letzte Fallbeispiel mit dem (besonders auf dem Feld der Kinder- und Jugendliteratur) etwas verstörenden Titel Durch Protektion (1931 von Varvara Cechovskaja in der Pariser Kinderzeitschrift Ogon 'ki publiziert). Das entwickelte, gelebte Demokratieverständnis des Editorial Board des Periodikums kommt dadurch zum Tragen, dass mittels gezielter redaktioneller Aufrufe an die junge Leserschaft Feedback eingefordert wurde; die Blattlinie sollte so aktiv mitgestaltet werden. Dem drohenden Identitätsverlust in der Emigration wurde durch den Abdruck ausgewählter Erwachsenenliteratur entgegengewirkt. Der Protagonist von Cechovskajas Erzählung ist Exponent eines neuen (nachrevolutionären) Erziehungsideals, das Mitverantwortung und aktives Engagement über passiven Konformismus stellt. Er wird aufgrund seiner zutiefst optimistischen Grundhaltung zum Hoffnungsträger einer neuen Generation, ist es doch - unter Auslotung der Rollenumkehr - keineswegs Standard, dass ein Kind sich für eine junge Erwachsene einsetzt, die es aufgrund ihres Exilantinnenstatus nicht wagen würde, sich um eine Stelle zu bewerben, für die sie geradezu ideal qualifiziert ist. Es ist ein erfindungsreiches - literaturtheoretisch nicht uninteressantes - Detail, dass der Bub die fragliche Stellenanzeige nur liest, weil ihm sein Vater ein Buch in gerade das Stück einer Zeitung eingeschlagen hat, auf dem die Annonce abgedruckt ist.

Das unbestreitbare Verdienst der Verfasserin ist eine reichhaltige Synopse auf Basis arbeitsintensiver Bibliotheks- und Archivarbeit, wobei sie auch den sozialwissenschaftlichen Aspekt nicht vernachlässigt: Preindl berichtet von (bestürzenden) Umfragen, die den Verlust der Kindheit und das aufgrund der Umstände zu schnelle Erwachsenwerden vieler Betroffener belegen. Sie setzt sich detailliert mit Qualitätskriterien auseinander, die in auch und gerade im Exil - intensiv geführten literaturtheoretischen Diskursen entwickelt wurden: Evgenij Elačič legte in seinen Untersuchungen (1921-1926) größten Wert auf die Verbreitung von Büchern, die Kinder intellektuell, ästhetisch und ethisch forderten und förderten. In Prag bewertete 1926-1928 die Kommission für Fragen der Kinderliteratur die im Exil kursierenden Werke, im Russischen Pädagogischen Museum in Paris sammelte die Fürstin Varvara Bobrinskaja 1929-1934 während ihrer Direktionszeit eine Fülle von Büchern und gründete eine Bibliothek für junge LeserInnen. Statistisch gesehen publizierten die meisten der die Exilliteratur prägenden AutorInnen Erwachsenen- sowie Kinder- und Jugendliteratur, was den (schrittweisen) Einstieg in die sogenannte Allgemeinliteratur für das junge Publikum fast mühelos ermöglichte. - Nadia Preindl hat ein reichhaltiges Repertorium geschaffen, das jede Möglichkeit zu eigenen Forschungen eröffnet. Ihre Musterinterpretationen regen zur Lektüre der Originale an und zum Vergleich mit dem, was sie aufgrund der ungeheuren Materialfülle nicht in derselben Intensität vorstellen konnte. Die Monographie ist ein Referenzwerk für alle, die Literatur und Literaturwissenschaft lieben und gleichzeitig verstehen wollen, wie Menschen jeglichen Alters und welcher Herkunft auch immer Flucht und Vertreibung, den Verlust der alten und das Finden einer neuen Heimat bewältigen - in der Wirklichkeit und in der Fiktion. Gerade das 20. Jahrhundert hat die Menschheit gelehrt, dass beides oft näher beieinander liegt, als man je geglaubt hätte. 